\title{
PHACOEMULSIFICATION IN DIABETICS
}

\author{
R. J. ANTCLIFF, A. POULSON and D. W. FLANAGAN \\ Cambridge
}

\begin{abstract}
SUMMARY
In a retrospective study we examined a consecutive group of diabetic patients (74 operated eyes) who underwent phacoemulsification and intraocular lens implantation over a 2 year period ending in June 1994. We compared this group with 66 diabetic eyes who underwent extracapsular surgery and lens implantation and who were examined for a previous study. There were no significant differences in progression of the retinopathy, complications, or final visual acuity. Seventy-two per cent of the phacoemulsification group improved by at least 2 lines of Snellen acuity postoperatively compared with $76 \%$ of the extracapsular group. Seventy-four per cent of the phacoemulsification group achieved an acuity of $6 / 12$ or better compared with $68 \%$ of the extracapsular group. Overall there were fewer post-operative complications in the phacoemulsification group though there was an increased incidence of transient corneal oedema. The major cause of poor visual acuity in the phacoemulsification group was maculopathy, particularly in the presence of proliferative retinopathy in older patients. Use of a small intraocular lens did not prevent adequate fundal examination or photocoagulation. It is concluded that the outcome of cataract surgery in diabetics is largely determined by the degree of maculopathy. Phacoemulsification and extracapsular cataract surgery give similar visual results. Diabetic retinopathy should not be considered a contraindication to small-incision cataract surgery and phacoemulsification.
\end{abstract}

Diabetes mellitus affects $2 \%$ of the population. ${ }^{1}$ Cataract occurs earlier in diabetics than non-diabetics $^{1,2}$ and therefore diabetics might benefit even more from the refractive advantages of phacoemulsification (decreased astigmatism and earlier visual rehabilitation). However, retinopathy also increases with age and duration of diabetes. ${ }^{3,4}$ Iris vessels have

From: Addenbrooke's Hospital and Hinchingbrooke Hospital, Cambridgeshire, UK.

Correspondence to: D. W. Flanagan, FRCOphth, Department of Ophthalmology, Clinic 3, Box 41, Addenbrooke's Hospital, Hills Road, Cambridge CB2 2QQ, UK. been shown to be more permeable in diabetics and diabetic iridopathy is usually associated with significant retinopathy. ${ }^{5}$ Diabetic eyes might therefore be expected to respond poorly to prolonged intraocular infusion and manipulation. Conversely phacoemulsification also minimises iris trauma as most surgical manoeuvres are behind an intact capsulorhexis and there is no need to express a nucleus through the pupil. Phacoemulsification on diabetics is not universally practised, but we now routinely carry out this procedure. This study reviews our results.

\section{PATIENTS AND METHODS}

The theatre records of all patients who underwent phacoemulsification under the care of one consultant surgeon over an 18 month period between January 1993 and June 1994 were examined. The medical coding records of these patients were then examined and the records of those patients who were diabetic were extracted for detailed examination. The patients were then recalled and examined by one of the authors. Seventy-five patients were included. Ten patients had died over the follow-up period; two of these patients who had been seen in the clinic with adequate follow-up (minimum of 6 months) were included. Four patients were lost to follow-up. Eleven patients had both eyes operated on. Sixtyone patients (72 eyes) were re-examined by one of the authors. A total of 63 patients ( 74 eyes) were therefore included.

All patients underwent phacoemulsification with implantation of a posterior chamber lens. Fifty eyes had a $5 \mathrm{~mm}$ optic, 7 had a $6 \mathrm{~mm}$ optic and 17 had a $6.5 \mathrm{~mm}$ optic implanted. Fifty-seven operations were carried out by one consultant surgeon and 17 by six different trainees.

Thirty of the eyes were from men and 44 from women. The ages ranged from 31 to 88 years (mean 67 years). The mean duration of follow-up was 12 months. Two patients were controlled by diet, 36 by oral hypoglycaemic agents, 35 were on insulin (11 of whom had originally commenced on oral

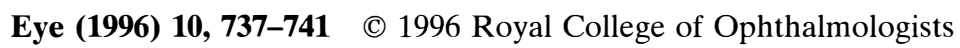




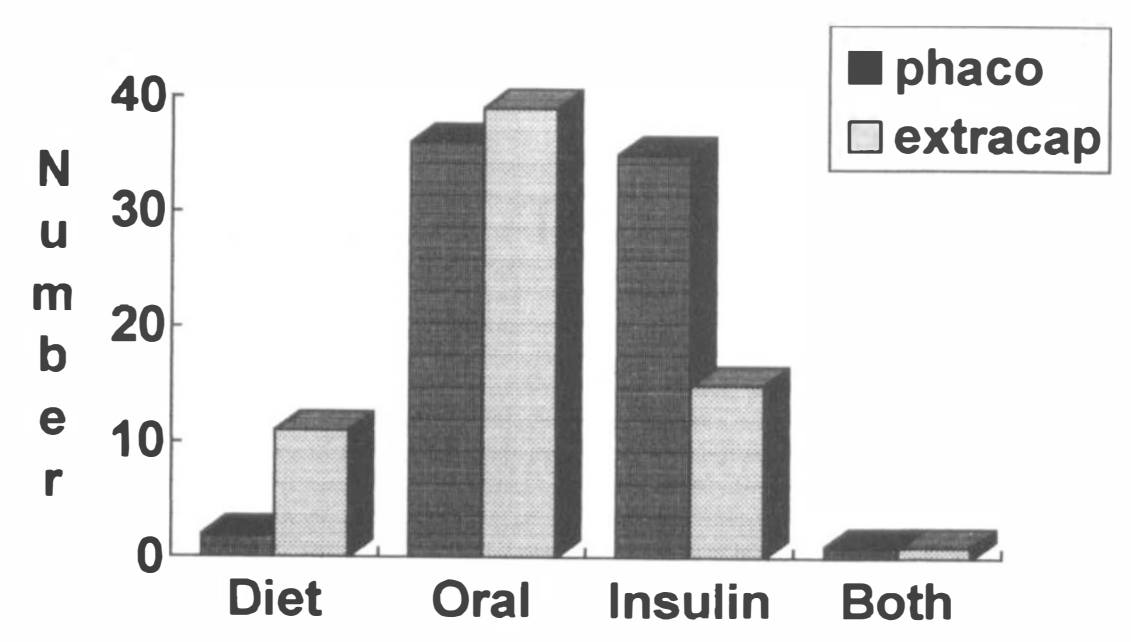

Fig. 1. The relative distribution of the treatments for diabetes in the patients undergoing phacoemulsification or extracapsular cataract extraction.

hypoglycaemic agents), and 1 patient was on both oral hypoglycaemic agents and insulin. The duration of disease in the oral hypoglycaemic group ranged from 1 to 43 years (mean 10.1 years) and in the insulin-dependent group ranged from 7 to 40 years (mean 23.3 years).

These patients were then compared with a similar group of diabetics who underwent extracapsular cataract extraction and who were examined for a previous study. ${ }^{6}$ The extracapsular eyes were from men in 28 cases and women in 38 cases (chi-squared $=1.28, p>0.1$. Six patients had both eyes operated on. The mean age was 70.5 years and the mean duration of follow-up was longer, at 2.61 years. Eleven patients were controlled by diet, 39 by oral hypoglycaemics, 15 were on insulin and 1 required both insulin and oral hypoglycaemics (Fig. 1).

\section{Visual}

\section{RESULTS}

There was no statistically significant difference in visual outcome between the two groups. In the phacoemulsification group $74 \%$ achieved a visual acuity of $6 / 12$ or better compared with $68 \%$ of the extracapsular group (chi-squared $=0.38, p>0.5$; Fig. $2)$. Seventy-two per cent of the phacoemulsification group improved by 2 or more lines of Snellen visual acuity compared with $76 \%$ of the extracapsular group (chi-squared $=0.13, p>0.5$ ). There was no difference in visual outcome between those on insulin and those on oral hypoglycaemic agents.

As with the previous study, the visual results were poorest in those with maculopathy or proliferative retinopathy (Table I). Sixteen eyes out of 31 with maculopathy improved by 2 or more lines (Fig. 3) and 16 eyes out of 27 with proliferative retinopathy improved by 2 lines.

Nineteen eyes failed to achieve $6 / 12$ at the followup visit. One patient had age-related macular degeneration and the other 18 all had maculopathy at final follow-up (Table II). Six eyes had severe

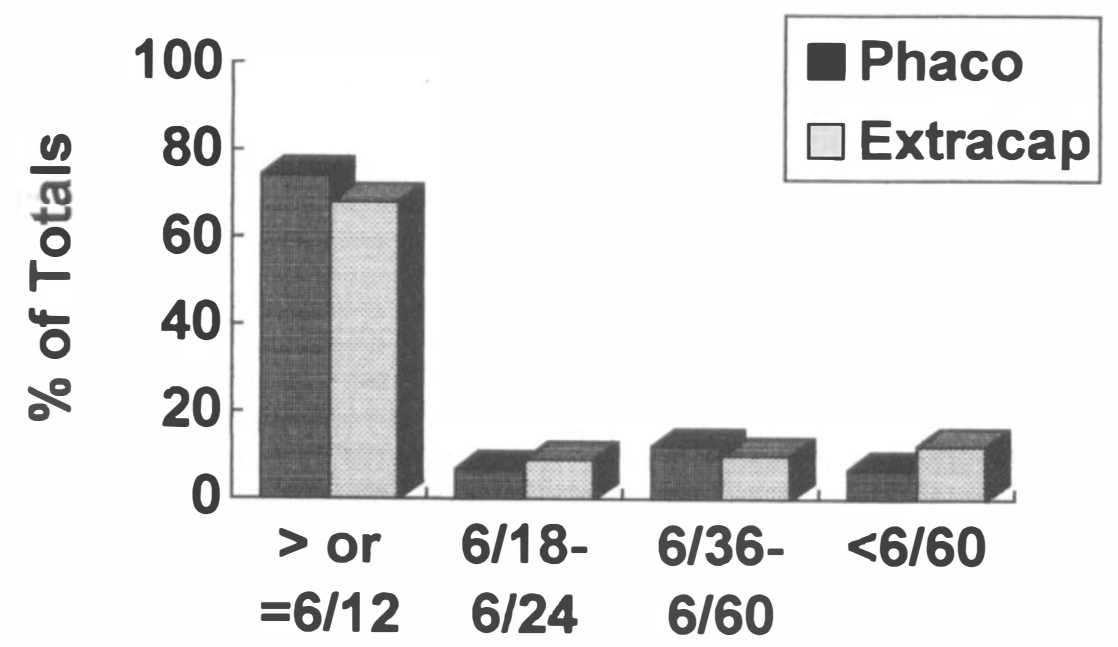

Fig. 2. The final visual acuities in the patients undergoing phacoemulsification or extracapsular cataract extraction. 
Table I. Final visual outcome after phacoemulsification

\begin{tabular}{|c|c|c|c|c|c|}
\hline & \multicolumn{5}{|c|}{ Final visual acuity: no. of eyes (\%) } \\
\hline & $\geqslant 6 / 12$ & $6 / 18-6 / 24$ & $6 / 36-6 / 60$ & $<6 / 60$ & Totals \\
\hline No retinopathy. & $20(83)$ & $1(4.2)$ & $2(8.4)$ & $1(4.2)$ & 24 \\
\hline \multicolumn{6}{|l|}{ Background retinopathy } \\
\hline With maculopathy & 10 & 2 & 2 & 0 & \\
\hline Without maculopathy & $9(82.6)$ & $0(8.7)$ & $0 \quad(8.7)$ & 0 & 23 \\
\hline \multicolumn{6}{|l|}{ Proliferative retinopathy } \\
\hline With maculopathy & 7 & 2 & 5 & 3 & \\
\hline Without maculopathy & $9(59.3)$ & $0(7.4)$ & $0(18.5)$ & $1(14.8)$ & 27 \\
\hline
\end{tabular}

chronic macular oedema, all with visual acuities of $6 / 60$ or worse.

\section{Progression of Retinopathy}

Twenty-five eyes showed progression of retinopathy post-operatively (Table III). Twenty-three eyes required photocoagulation post-operatively. Two eyes had panretinal photocoagulation (PRP), 12 eyes had macular treatment and 9 eyes had both PRP and macular laser treatment.

\section{Complications}

There were few peroperative complications. There were 3 capsule ruptures, 2 requiring anterior vitrectomies. On 2 occasions the capsulorhexis was converted to a capsulotomy and twice the capsulorhexis was torn. There were 2 zonular dehiscences and 1 iris tear. This compares with no peroperative complications in the extracapsular group.

The most common post-operative complication was capsular thickening. This occurred in $20(27 \%)$ of the phacoemulsification group (Table IV) and 23 $(35 \%)$ of the extracapsular group (chi-squared = $0.67, p>0.1)$. The mean diameter of the capsulorhexis was $4.6 \mathrm{~mm}$ (range $3-7 \mathrm{~mm}$ ), and the mean pupil diameter was $6.7 \mathrm{~mm}$ (range 4-9 mm) 30 minutes after installation of $10 \%$ phenylephrine and $1 \%$ cyclopentalate.
In the phacoemulsification group 16 eyes had the optic only partially inside the bag (both haptics were in the bag), but none of these optics was displaced (Fig. 4). Overall there were fewer postoperative complications in the phacoemulsification group, but 8 eyes had transient corneal oedema and 8 eyes had transiently raised intraocular pressure $(>25 \mathrm{mmHg})$, whereas none of the extracapsular eyes suffered from this complication.

\section{DISCUSSION}

The phacoemulsification group had a greater proportion of insulin-dependent diabetics to non-insulindependent diabetics than the extracapsular group previously reported ${ }^{6}$ (Fig. 1). There was also a greater proportion of diabetics with proliferative retinopathy preoperatively (chi-squared $=8.12$; $0.02>p>0.01$ ), and a greater proportion of diabetics with maculopathy (chi-squared $=2.1 ; p>0.1$ ). The duration of follow-up was also shorter in the phacoemulsification group (12 months vs 2.61 years). This implies that the results must be interpreted with caution.

There have been several published series of extracapsular cataract extraction in diabetics, ${ }^{7-12}$ but few of phacoemulsification. ${ }^{13}$ Lack of universal acceptance of phacoemulsification may be attributed to difficulty photocoagulating through a small optic

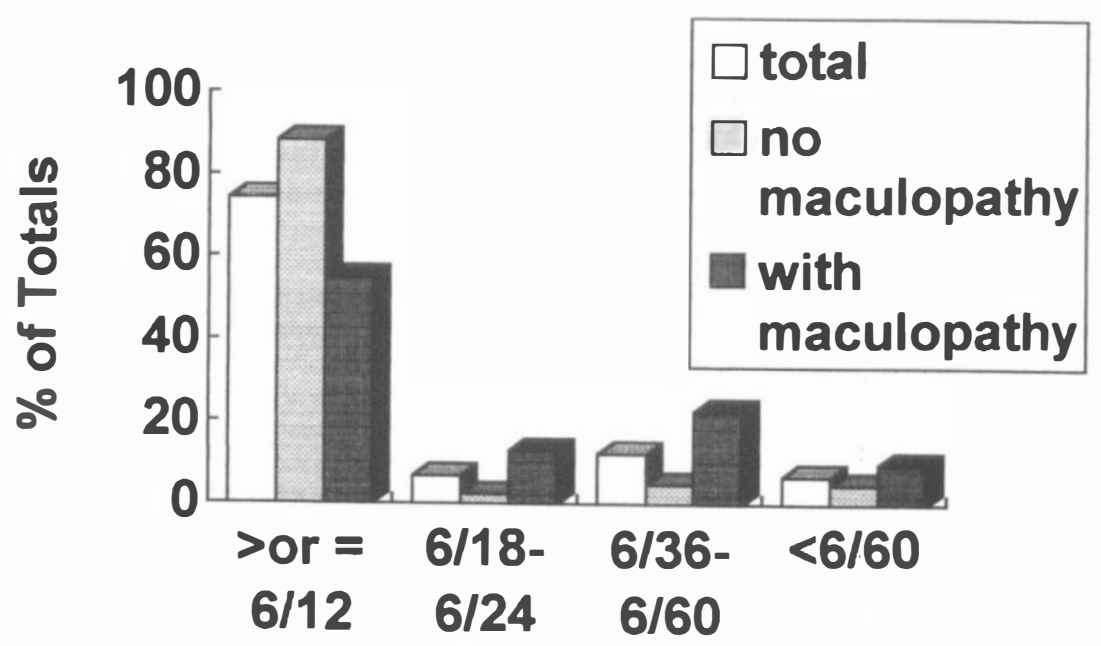

Fig. 3. The final visual acuities of the phacoemulsification patients with and without maculopathy. 
Table II. Preoperative retinopathy in those failing to achieve a post-operative visual acuity of $6 / 12$ or better after phacoemulsification

\begin{tabular}{lcc}
\hline & No maculopathy & Maculopathy \\
\hline No retinopathy & 3 & 0 \\
Background & 0 & 4 \\
Proliferative & 1 & 10 \\
\hline
\end{tabular}

Table III. Progression of existing retinopathy or development of new grade of retinopathy post-operatively in phacoemulsifcation patients

\begin{tabular}{lcccc}
\hline \multirow{2}{*}{$\begin{array}{l}\text { Preoperative } \\
\text { retinopathy }\end{array}$} & Background & New & Existing & Proliferative \\
\cline { 3 - 5 } & 1 & 3 & - & 1 \\
None & - & 3 & 7 & 3 \\
Background & - & - & 2 & 5 \\
\hline
\end{tabular}

and a thickened anterior capsule, as well as to a presumed higher rate of surgical complications compared with that in extracapsular extraction.

Photocoagulation was difficult through the smaller optics but it was possible to treat all eyes adequately. No eyes in the phacoemulsification group developed rubeosis. At final follow-up 30 eyes had adequately treated proliferative retinopathy, compared with 15 of the extracapsular group. Eighteen of these (60\%) achieved a visual acuity of $6 / 12$ or better, the same proportion as the extracapsular group. This compares with $28-48 \%$ of patients with proliferative retinopathy in previous studies. ${ }^{7.11}$ The main cause of poor visual acuity post-operatively was preoperative maculopathy, particularly when combined with preoperative proliferative retinopathy. This confirms previous work..$^{6,8,12}$ Seven eyes out of 17 (41\%) with proliferative diabetic retinopathy (PDR) and maculopathy achieved a visual acuity of $6 / 12$ or better, whereas 9 eyes out of $10(90 \%)$ with PDR without

Table IV. Post-operative complications

\begin{tabular}{lcc}
\hline Complication & $\begin{array}{c}\text { Phaco- } \\
\text { emulsification }\end{array}$ & $\begin{array}{c}\text { Extra- } \\
\text { capsular }\end{array}$ \\
\hline Capsular thickening & 20 & 23 \\
Transient corneal oedema & 8 & 0 \\
Transient raised pressure & 8 & 0 \\
Pseudophakic cystoid macular oedema & 3 & 4 \\
Hyphaema & 2 & 0 \\
Synechiae to capsule & 2 & 0 \\
Synechiae to IOL & 1 & 9 \\
Uveitis & 1 & 4 \\
Abrasion & 1 & 0 \\
Sterile hypopyon & 0 & 1 \\
Fungal keratitis & 0 & 1 \\
IOL malposition & 0 & 2 \\
Iris prolapse & 0 & 1 \\
Corneal decompensation & 0 & 1 \\
Endophthalmitis & 0 & 1 \\
Suture infiltrate & 0 & 1 \\
Vitreous haemorrhage & 1 & 4 \\
Rubeosis and neovascular glaucoma & 0 & 4 \\
Totals & 47 & 56 \\
\hline
\end{tabular}

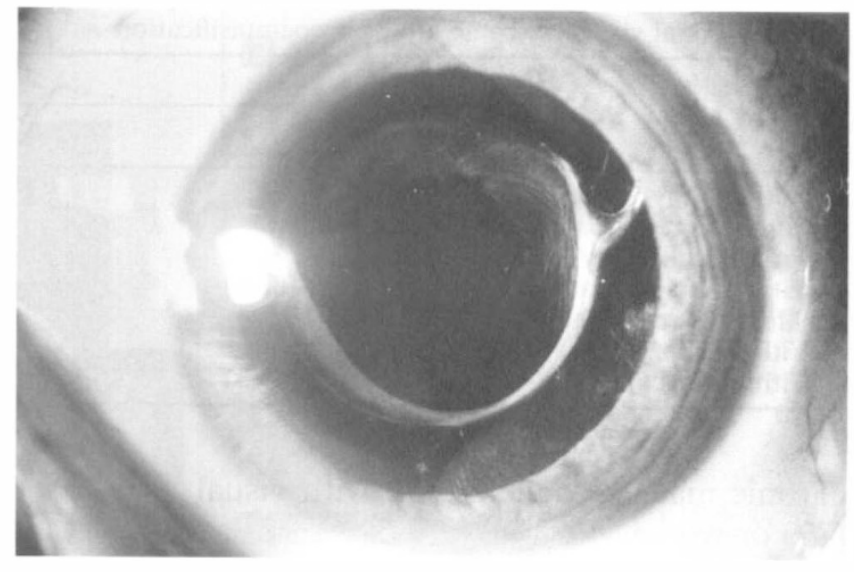

Fig. 4. An optic only partially inside the capsulorhexis, but with both haptics in the bag.

maculopathy achieved $6 / 12$ or better. The mean age of those with PDR without maculopathy was 51 years and of those with PDR with maculopathy was significantly older at 64 years $(0.05>p>0.01$ by $t$ test). This confirms that older patients with PDR and maculopathy have the worst prognosis and therefore surgery in this group needs detailed preoperative assessment and careful justification.

The rate of serious complications was small in both groups, but lower in the phacoemulsification group; only 1 eye had an adhesion between the iris and the implant. The capsulotomy rate was comparable to previously reported rates of $21.8-28 \% .^{14.15}$ The final visual acuities were no different and compare favourably with previous reported series where $10-48 \%$ achieved $6 / 12$ or better. ${ }^{7.8,10,11}$

Small incision cataract surgery and phacoemulsification give similar visual results to conventional extracapsular cataract surgery in diabetics with and without retinopathy. Reduced post-operative intraocular inflammation, fewer iris-intraocular lens adhesions and a secure wound facilitate early postoperative observation and photocoagulation. Small optics and a preserved anterior capsule do not prevent adequate photocoagulation though they can make it more difficult. Diabetic retinopathy should therefore not be considered a contraindication to small incision surgery and phacoemulsification.

Key words: Diabetes, Retinopathy, Cataract, Phacoemulsification, Extracapsular extraction.

\section{REFERENCES}

1. Weatherall DJ, Ledingham JGG, Warrell DA. Oxford textbook of medicine, vol 1. 2nd ed. Oxford: Oxford University Press, 1987: chap 9.

2. Benson WE. Cataract surgery and diabetic retinopathy. Curr Opin Ophthalmol 1992;3:396-400.

3. Klein R, Klein BEK, Moss SE, Cruickshanks KJ. The Wisconsin Epidemiologic Study of Diabetic Retinopathy. XIV. Ten-year incidence and progression of 
diabetic retinopathy. Arch Ophthalmol 1994; 112:1217-28.

4. Nathan DM. Long-term complications of diabetes mellitus. N Engl J Med 1993;328:1676-?.

5. Bandello F, Brancato R, Lattanzio R, Galdini M, Falcomata B. Relation between iridopathy and retinopathy in diabetes. Br J Ophthalmol 1994;78:542-5.

6. Cunliffe IA, Flanagan DW, George NDL, Aggarwaal RJ, Moore AT. Extracapsular cataract surgery with lens implantation in diabetics with and without proliferative retinopathy. $\mathrm{Br} \mathrm{J}$ Ophthalmol 1991;75:9-12.

7. Hykin PG, Gregson RMC, Stevens JD, Hamilton AM. Extracapsular cataract extraction in proliferative diabetic retinopathy. Ophthalmology 1993;100:394-9.

8. Benson WE, Brown GC, Tasman W, McNamara JA, Vander JF. Extracapsular cataract extraction with placement of a posterior chamber lens in patients and diabetic retinopathy. Ophthalmology 1993;100:730-8.

9. Ruiz RS, Saatchi OA. Posterior chamber intraocular lens implantation in eyes with inactive and active proliferative diabetic retinopathy. Am J Ophthalmol 1991;111:158-62.
10. Jaffe GJ, Burton TC, Kuhn E, Prescott A, Hartz A. Progression of nonproliferative diabetic retinopathy and visual outcome after extracapsular cataract extraction and intraocular lens implantation. Am J Ophthalmol 1992; 114:448-56.

11. Schatz H, Atienza D, McDonald HR, Johnson RN. Severe diabetic retinopathy after cataract surgery. Am J Ophthalmol 1994;117:314-21.

12. Dowler JGF, Hykin PG, Lightman SL, Hamilton AM. Visual acuity following extracapsular cataract extraction in diabetes: a meta analysis. Eye 1995;9:313-7.

13. Fung WE. Phacoemulsification and implantation of posterior chamber intraocular lens in eyes with quiescent proliferative diabetic retinopathy. Graefes Arch Clin Exp Ophthalmol 1987;225:251-3.

14. Ionides A, Dowler JGF, Hykin PG, Rosen PH, Hamilton AM. Posterior capsule opacification following diabetic extracapsular cataract extraction. Eye 1994;8:535-7.

15. Knorz MC, Soltau JB, Seiberth V, Lorger C. Incidence of posterior capsule opacification after extracapsular cataract extraction in diabetic patients. Metab Pediatr Syst Ophthalmol 1991;14:35-6. 